\title{
Relations between serum and pleural fluid biomarkers: a new look of an old concept
}

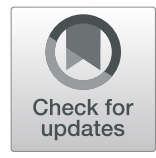

Sabah Ahmed Hussein ${ }^{1 *}$ and Maha Yousif Elhefnawy ${ }^{2}$

\begin{abstract}
Background: Although cytological examination of pleural fluid samples can simply diagnose malignant pleural effusion (MPE), this test has many limitations. There are no established biomarkers for accurate diagnosis of MPE. This study investigated the association of serum lactate dehydrogenase (LDH)/pleural fluid adenosine deaminase (ADA) which is the cancer ratio with MPE together with assessment of the utility of combining pleural lymphocyte counts "cancer ratio plus" in diagnosing MPE and to evaluate the ability of these markers in differentiating MPE from tuberculous pleural effusion (TPE).

Results: This prospective study included 150 individuals who were divided into 3 groups including malignant $(n=$ 94), tuberculous $(n=31)$, and parapneumonic $(n=25)$ effusions. It was done during the period from January 2018 to July 2019 to assess the utility of cancer ratio and cancer ratio plus in discrimination between MPE and non-MPE. Serum LDH, cancer ratio, and cancer ratio plus were significantly associated with MPE. Also, age, cancer ratio, and serum LDH to pleural fluid lymphocyte count ratio were positive predictors of MPE. A cutoff level of $>16.02$ for the cancer ratio showed sensitivity and specificity of $61 \%(95 \% \mathrm{Cl} 0.5002-0.7056)$ and $96 \%(95 \% \mathrm{Cl} 0.8769-0.9956)$ respectively. At this cutoff, the positive likelihood ratio was 16.99, while the negative likelihood ratio was 0.41 .

Conclusion: Cancer ratio was found to be more accurate than cancer ratio plus and serum LDH to pleural fluid lymphocyte count ratio in identifying MPE especially in patients with negative pleural fluid cytology.

Keywords: Cancer ratio, Cancer ratio plus, Pleural effusion
\end{abstract}

\section{Background}

Pleural effusion is one of the common clinical entities. Many diseases may be associated with pleural effusion formation including local conditions affecting the pleura and secondary pleural affection from extrapulmonary diseases. Differentiation between exudate and transudate is the common initial diagnostic step to patients with pleural effusion [1]. In clinical practice, the most common three conditions resulting in exudative effusion are tuberculous pleural effusion (TPE), malignant pleural effusion (MPE), and parapneumonic pleural effusion (PPE). More analysis of pleural fluid, including total and differential cell count, $\mathrm{pH}$, glucose levels, adenosine deaminase (ADA) activity, and cytological and microbiological examinations, is

\footnotetext{
* Correspondence: Sabah.Hussein@kasralainy.edu.eg

${ }^{1}$ Faculty of Medicine, Cairo University, Cairo, Egypt

Full list of author information is available at the end of the article
}

required for further differentiation between the specific diseases associated with formation of pleural exudate [2]. A high neutrophil count in the pleural fluid is indicative of parapneumonic effusion, and a high level of pleural fluid ADA level is mostly suggestive (specificity of 92\%) for tuberculosis (TB), while regarding MPE, to date, no test is specific to "rule-in" MPE [3].

Although MPE can be simply diagnosed by pleural fluid cytological analysis, this test has many limitations, including variability in the sensitivity of the test which is ranging from 11.6 to $71 \%$ [4]. There are no established accurate biomarkers for the diagnosis of MPE. Most tumor markers were not sensitive nor specific to be implemented in routine assessment of MPE. Data are insufficient on the true diagnostic performance of the relationship between low ADA levels and MPE [5, 6]. Due to the limitations of noninvasive diagnostic tests of 
MPE, many patients may require more invasive diagnostic tests with associated complications and some delay in the cancer diagnosis.

The "cancer ratio" (CR) which is a quotient of serum lactate dehydrogenase (LDH) and pleural fluid ADA is a new biochemical parameter that showed a high diagnostic accuracy for pleural malignancies. Cancer ratio showed high diagnostic value based on the observations that MPE is often associated with high serum LDH levels and comparatively low pleural fluid ADA levels and lymphocyte count $[7,8]$. On the other hand, TPE patients had low LDH in the serum with high pleural ADA levels and also raised pleural fluid lymphocyte counts. These relations between serum and pleural fluid biomarkers resulted in the development of a ratio with the diagnostic value to help differentiating MPE from TPE in a cost-effective and clinically applicable manner. These markers may give early signal toward the possibility of the malignant nature of pleural fluid and can potentially serve as a "forewarning" for patients with negative cytology who may subsequently have MPE [8].

This study was aiming to investigate the association between the cancer ratio and MPE associated with the assessment of the utility of combining pleural lymphocyte counts "cancer ratio plus" in diagnosing MPE and to study the ability of these markers in differentiating MPE from tuberculous pleural effusion (TPE).

\section{Methods}

This cross-sectional study included 150 patients with exudative pleural effusion who were recruited from the chest departments of Cairo University and Menoufia University hospitals during the period from January 2018 to July 2019. Institutional ethical committee approval and patients' written informed consents were obtained before enrollment. All studied patients were subjected to full history talking, complete clinical examination, and routine serum biochemical analysis including serum protein and serum LDH and chest X-ray. Chest computed tomography and/or transthoracic ultrasound were done whenever indicated. Pleural fluid aspiration was done, and pleural fluid was sent for protein level, LDH, ADA, microbiology, and cytology. Light's criteria were used to classify effusions as transudates or exudates [9]. Thoracoscopic or ultrasound-guided closed pleural biopsy was also obtained to confirm the diagnosis. Clinical and laboratory data of patients with MPE, TPE, and PPE were prospectively analyzed. Exudative effusions other than the previously mentioned groups and patients with pleural transudates were excluded from the study.

Three ratios were calculated and analyzed including: a) "Cancer ratio" which is the ratio between serum LDH and pleural ADA [7].

b) "Cancer ratio plus" which is the ratio of cancer ratio to the percentage of differential pleural lymphocyte count. It was calculated to see the effect of pleural lymphocyte count and cancer ratio on the accuracy of identifying MPE [8].

c) The ratio between serum LDH and pleural lymphocyte count. It was calculated to see the effect of serum LDH and pleural lymphocyte count on the accuracy of identifying MPE [8].

\section{Statistical methods}

The data were collected, tabulated, and statistically analyzed using the Minitab 17.1.0.0 for windows (Minitab Inc., 2013, PA, USA). All tests were two sided. A $p$ value $<0.05$ was considered significant. Continuous data were represented as mean and SD or median and IQR, and categorical data as number and percentage. One-way ANOVA test with post hoc analysis using Tukey or Dunnt's methods was used to compare between more than groups of numerical data nature. Multiple logistic regressions were used to estimate the most independent predictors for malignant outcome; furthermore, ROC analysis was performed using SigmaPlot software 12.5.0.38 for windows (SigmaPlot, Systat Software Inc. UK, 2011) to evaluate the performance of significant predictors besides the determination of best cutoff points.

\section{Results}

One hundred and fifty patients with exudative pleural effusion were included in the study; demographic and pleural fluid characteristics of all patients are summarized in Table 1 . The frequency of malignant pleural effusion was $62.67 \%$ (94 patients); on the other hand, tuberculous and para-pneumonic effusions were $20.67 \%$ (31 patients) and $16.67 \%$ (25 patients) respectively. Comparison between the different characteristics of patients with MPE, TPE, and PPE is shown in Table 2. Older age was significantly reported in malignant patients than in TB and para-pneumonic groups $(P<0.001)$. The pleural fluid ADA and lymphocyte count were significantly higher in TB effusion than in MPE $(P<0.001$ for both). Furthermore, the serum LDH was significantly elevated in MPE than in TPE and PPE even with insignificant difference between its level in pleural fluid within the groups $(P<0.001$ and 0.89 respectively). Moreover, to explore the more potency of combining LDH level in the serum with ADA level in the pleural fluid, the cancer ratio was calculated and showed significant elevation in MPE than in both inflammatory groups, i.e., TPE and PPE $(P<0.001)$, while the cancer ratio plus and the ratio between serum LDH and lymphocyte count 
Table 1 Demography and pleural fluid characteristics of the studied patients

\begin{tabular}{|c|c|}
\hline Variable & Total $(n=150)$ \\
\hline Age (years) (mean $\pm \mathrm{SD}$ ) & $53.63 \pm 13.57$ \\
\hline Sex (male) (number, \%) & $71,47.33 \%$ \\
\hline Smoking history (yes) (number, \%) & $58,38.67 \%$ \\
\hline Pleural fluid glucose (g/dl) (mean $\pm \mathrm{SD}$ ) & $93.01 \pm 48.91$ \\
\hline Pleural fluid protein (g/dl) (mean $\pm \mathrm{SD}$ ) & $4.75 \pm 1.04$ \\
\hline Serum protein $(\mathrm{g} / \mathrm{dl})($ mean $\pm \mathrm{SD})$ & $6.45 \pm 0.86$ \\
\hline Pleural fluid/serum protein (mean \pm SD) & $0.75 \pm 0.19$ \\
\hline Pleural fluid LDH (U/I) (mean \pm SD) & $720.70 \pm 523.20$ \\
\hline Serum LDH (U/I) (mean \pm SD) & $312.70 \pm 141.80$ \\
\hline Pleural fluid LDH/Serum LDH & $2.78 \pm 2.17$ \\
\hline Pleural fluid ADA (U/I) (mean \pm SD) & $24.63 \pm 12.43$ \\
\hline Cancer ratio (mean $\pm \mathrm{SD}$ ) & $17.02 \pm 13.46$ \\
\hline Pleural fluid lymphocyte count (\%) (mean \pm SD) & $0.52 \pm 0.24$ \\
\hline Cancer ratio plus (mean $\pm \mathrm{SD}$ ) & $47.44 \pm 62.09$ \\
\hline $\begin{array}{l}\text { Serum LDH/pleural fluid lymphocyte } \\
\text { count (mean } \pm \text { SD) }\end{array}$ & $847.10 \pm 816.10$ \\
\hline
\end{tabular}

$L D H$ lactate dehydrogenase, $A D A$ adenosine deaminase, $g / d l$ grams per deciliter, U/I units per liter

in the pleural fluid showed significant elevation in the malignant and para-pneumonic groups than the TB group of pleural effusion $(P<0.001)$. Elevated cancer ratio was found to be more accurate than cancer ratio plus and serum LDH and lymphocyte count ratio in identifying MPE.

Multivariate logistic regression analysis that was adjusted using stepwise elimination methods for all predictors of malignant effusion still pointed on age, "cancer ratio," and "serum LDH to pleural lymphocyte count" as significant positive predictors of MPE (Table 3). The performance of predictors was clarified in the ROC curve (Fig. 1); the AUC of cancer ratio and age suggested good accuracy in discriminating MPE $(88 \%$ and $84 \%$ respectively) with $P<0.001$ for both, while AUC of serum LDH to pleural fluid lymphocyte ratio was fair to be accepted for discriminating MPF (AUC 65\% and $P=$ 0.001). Paired test of each ROC analysis showed that the difference of area between "cancer ratio" and "serum LDH to pleural fluid lymphocyte ratio" was significant, the same for the difference of area between age and "serum LDH to pleural fluid lymphocyte ratio" $(P<0.0001$ and 0.0003 respectively), while the difference of area between cancer ratio and age was insignificant $(P=0.38)$ (Table 4). Again, this means that cancer ratio was more accurate than serum LDH and lymphocyte count ratio in identifying MPE. The sensitivity, specificity, LR+, LR-, PPV, and NPV of cancer ratio and age at different cutoff points are concluded in Table 5 .

\section{Discussion}

Considering the previously few published studies, this study was done to evaluate the diagnostic ability of recently mentioned "cancer ratio" and "cancer ratio plus" and to assess their usefulness as potential predictors discriminating MPE from other etiologies of pleural exudates. Regarding patient's demography and characteristics of the pleural fluid among the study patients (Table 1), the mean age was 53.63 with \pm SD 13.57, males were 71 (47.33\%), and females were 79 (52.67\%); 58 (38.765) patients were smokers, and the mean values of pleural fluid glucose, protein, LDH, and ADA were 93.01, 4.75, 720.70, and 24.63 respectively. Also, the mean values of "cancer

Table 2 Univariate analysis between the different types of pleural effusion

\begin{tabular}{|c|c|c|c|c|c|c|}
\hline Variable & Malignant $(n=94)$ & Tuberculous $(n=31)$ & Para-pneumonic $(n=25)$ & P1 & P2 & P3 \\
\hline Age & $59.97(9.25)$ & $38.03(10.8)$ & $49.16(13.18)$ & $<0.001$ & $<0.001$ & $<0.001$ \\
\hline Pleural fluid glucose & $90(75-109.25)$ & $87(60-100)$ & $70(52.5-90.5)$ & 0.5 & 0.01 & 0.1 \\
\hline Pleural fluid protein & $4.67(0.9)$ & $4.84(1.2)$ & $4.96(0.9)$ & & $0.41 *$ & \\
\hline Serum protein & $6.45(5.9-7)$ & $6.5(5.8-7.1)$ & $6.5(5.8-7)$ & & $0.99 *$ & \\
\hline Pleural fluid/serum protein & $0.71(0.61-0.82)$ & $0.77(0.64-0.9)$ & $0.76(0.61-0.88)$ & & $0.34^{*}$ & \\
\hline Pleural fluid LDH & 555 (355.75-1011) & $600(299-844)$ & $469(321-1046)$ & & $0.89 *$ & \\
\hline Serum LDH & 342.5 (233.75-420) & $220(189-276)$ & $220(190-259)$ & 0.001 & 0.001 & 0.9 \\
\hline Pleural fluid LDH/Serum LDH & $1.75(1.07-3.34)$ & $2.59(1.49-3.63)$ & $2.13(1.25-4.95)$ & & 0.06 & \\
\hline Pleural fluid ADA & $19(11-25)$ & $38(29-42)$ & $24(20-30)$ & $<0.001$ & 0.001 & $<0.001$ \\
\hline Cancer ratio & $18.03(10.87-29.73)$ & $6.1(4.64-9.17)$ & $8.7(6.79-11.79)$ & $<0.001$ & $<0.001$ & $<0.001$ \\
\hline Pleural fluid lymphocyte count & $0.5(0.4-0.6)$ & $0.88(0.8-0.95)$ & $0.2(0.15-0.3)$ & $<0.001$ & $<0.001$ & $<0.001$ \\
\hline Cancer ratio plus & $40.13(24.68-60.88)$ & $8.06(5.22-11.14)$ & $47.5(32.15-68.78)$ & $<0.001$ & 0.5 & $<0.001$ \\
\hline Serum LDH/pleural fluid lymphocyte count & $700(506.8-950.4)$ & $271.11(216.49-332.5)$ & 1200 (762.12-1754.54) & $<0.001$ & $<0.001$ & $<0.001$ \\
\hline
\end{tabular}

Normal distributed data represented as mean (SD) and non-normally distributed data as median (IQR); the test of significant is one-way ANOVA with multiple comparison using Tukey's methods or Dunnet's methods

$P 1$ malignant vs tuberculous, $P 2$ malignant vs para-pneumonic, $P 3$ tuberculous vs para-pneumonic, $P<0.05$ considered significant

*Not applicable post hoc test, because $P>0.05$ 
Table 3 Multivariate analysis of predictors used in malignancy

\begin{tabular}{|c|c|c|c|c|}
\hline Variable & Coefficient & Odd & $95 \% \mathrm{Cl}$ & $P$ \\
\hline Age & 0.11 & 1.12 & $(1.0577,1.1835)$ & $<0.001$ \\
\hline Pleural fluid glucose & 0.01 & 1.01 & $(0.9897,1.0339)$ & 0.28 \\
\hline Serum LDH & 0.01 & 1.01 & $(0.9940,1.0178)$ & 0.33 \\
\hline Pleural fluid ADA & 0.05 & 1.05 & $(0.9415,1.1651)$ & 0.40 \\
\hline Cancer ratio & 0.33 & 1.40 & $(1.0544,1.8485)$ & 0.007 \\
\hline Pleural fluid lymphocyte count & -3.93 & 0.02 & $(0.0004,1.0271)$ & 0.05 \\
\hline Serum LDH to pleural fluid lymphocyte count & 0.00 & 1.00 & $(0.9957,0.9991)$ & 0.003 \\
\hline
\end{tabular}

Person $X^{2}=162.02, P=0.12$, the model adjusted for all variables using stepwise elimination methods, the sign before the coefficient denote the direction of relationship, $P$ considered significant if $<0.05$

$\mathrm{Cl}$ confident interval

ratio," "cancer ratio plus," and "serum $\mathrm{LDH} /$ pleural fluid lymphocyte count" were 17.02, 47.44, and 847.10 respectively.

Univariate analysis between the 3 different etiological types of pleural effusion (Table 2) revealed statistically significant difference between the 3 study groups regarding the mean age with higher age among the malignant group (mean \pm SD; $59.97 \pm 9.25$ ) in comparison with lower age in the tuberculous (mean \pm SD; $38.03 \pm$ 10.8 ) and para-pneumonic groups (mean \pm SD; $49.97 \pm$ 13.18). There was statistically significant difference between MPE and TPE in serum LDH level $(\mathrm{P} 1=0.001)$ as

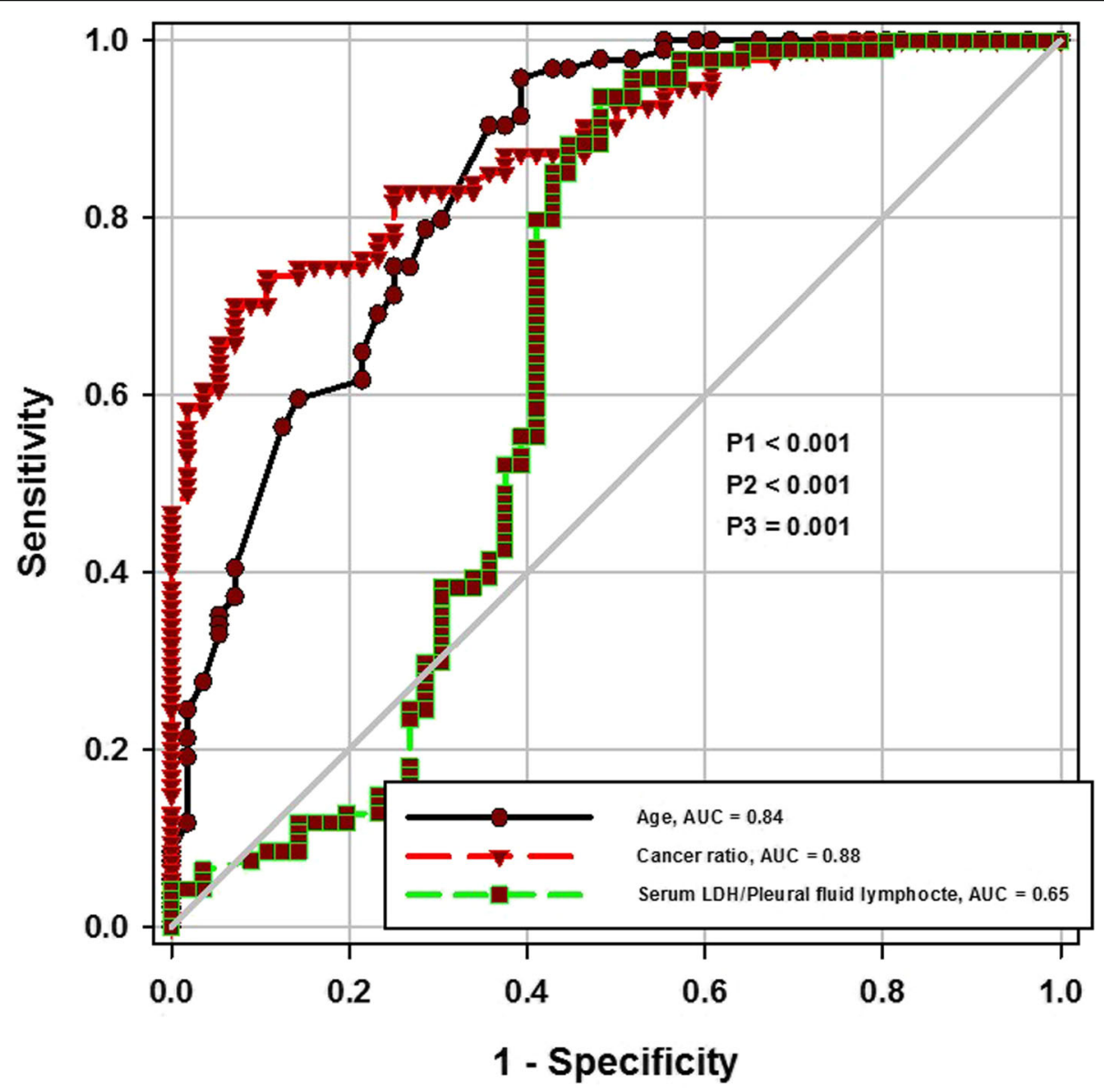

Fig. 1 ROC curve for age, cancer ratio and serum LDH to pleural fluid lymphocyte count ratio 
Table 4 Comparison between AUC of each predictor

\begin{tabular}{lll}
\hline Paired test & Area difference & $P$ \\
\hline $\begin{array}{l}\text { Cancer ratio vs serum LDH to pleural } \\
\text { fluid lymphocyte count }\end{array}$ & $\mathbf{0 . 2 3}$ & $<\mathbf{0 . 0 0 0 1}$ \\
$\begin{array}{l}\text { Age vs serum } L D H \text { to pleural fluid } \\
\text { lymphocyte count }\end{array}$ & $\mathbf{0 . 1 9}$ & $\mathbf{0 . 0 0 0 3}$ \\
Cancer ratio vs age & $-\mathbf{0 . 0 3}$ & $\mathbf{0 . 3 8}$ \\
\hline Test of significance is paired chi square test, $P$ considered significant if $<0.05$
\end{tabular}

it was higher in MPE (median/range; 342.5/233-420) in comparison with TPE (median/range; 220/189-276). Again, the difference between MPE and PPE regarding median level of serum LDH was statistically significant $(\mathrm{P} 2=0.001)$ as it was lower in PPE (median/range; 220/ 190-259) in comparison with MPE. The pleural fluid ADA median value showed statistically significant difference between the 3 groups with higher values in TPE (median/range; 39/29-42) and PPE (median/range; 24/ 20-30) than MPE (median/range; $19 / 11-25)(P<0.001)$.

Lactate dehydrogenase is a serum cellular enzyme that rises in a non-specific manner in response to tissue injury. High levels of serum LDH were found in many clinical conditions. The diagnostic and prognostic role has been studied previously, and it was reported as a poor prognostic marker in sepsis and malignancy [10]. The possible causes for its elevation in malignancies are mainly the use of glycolysis for energy by cancer cells, instead of oxidative phosphorylation, a switch in the adenosine triphosphate (ATP) generation pathway which is mediated by LDH. Growing cells needs a high rate of glycolysis because it is capable of producing ATP faster than oxidative phosphorylation. Glycolysis is better suited to meet the demand of growing cells as they need an enormous demand for ATP to fuel their growth [11]. The present study reported association between elevated serum LDH and MPE which is going with the previously mentioned relations between $\mathrm{LDH}$ in serum and malignancy [12].
Furthermore, ADA is produced by mononuclear cells, lymphocytes, neutrophils, and red blood cells. There are 2 subtypes of ADA, "ADA-1" and "ADA-2." High levels of ADA-2 correlate with TB, and high levels of ADA-1 are associated with empyema [13]. Malignant effusions show comparatively low levels of ADA. Such low levels cannot be used to diagnose malignant effusion due to the absence of biochemical relationship between them. On the other hand, many studies showed that serum $\mathrm{LDH}$ was high in malignancies with the well-studied mechanism [12]. So these two markers who showed negative and positive prediction of malignancy were combined in an attempt to develop a predictor of malignant pleural effusion. This ratio was significantly higher in the malignant group versus the $\mathrm{TB}$ and parapneumonic groups.

The highest median value of pleural fluid lymphocyte count was in the TPE group (median/range; 0.88/0.80.95 ) followed by the MPE group (median/range; 0.5/0.40.6 ), and the lowest value was in the PPE group (median/ range; $0.2 / 0.15-0.3$ ) with a statistical significant difference between the 3 study groups $(P<0.001)$ (Table 2$)$. This agrees with Verma et. al. [8] who reported that the median lymphocyte count was higher in TPE than MPE (86\% versus $61 \%, P<0.007)$ and agrees with previous studies [1416]. TPE have been shown to be associated with high percentages of lymphocytes in the pleural fluid. One study reported that $67 \%$ of patients with TPE have pleural lymphocyte percentage of $>95 \%$ [17]. Again, another study on TPE revealed that $>50 \%$ of leukocytes in pleural fluid were lymphocytes with mean \pm SD of $77 \pm 19.9$ and median (range) of 80.5 (2-100\%) [18]. Also, a larger study including 382 patients with TPE revealed that median lymphocyte percentage of total cells was $84 \%$ [19]. Lymphocyte predominance in $60-90 \%$ of cases of TPE was also described in several other studies [20]. Lymphocyte count of $<50 \%$ may occur in TPE exceptionally in only $\sim 5 \%$ [21]. So TPE represent the most frequent cause

Table 5 Sensitivity, specificity, PPV, and NPV of cancer ratio and age at different cutoff points

\begin{tabular}{|c|c|c|c|c|c|c|c|c|c|}
\hline & Cutoff points & Sensitivity (\%) & $95 \% \mathrm{Cl}$ & Specificity (\%) & $95 \% \mathrm{Cl}$ & LR+ & LR- & PPV(\%) & $N P V(\%)$ \\
\hline \multirow[t]{4}{*}{ Age } & $>39.5$ & 100 & 0.9615 to 1.000 & 45 & 0.3134 to 0.5853 & 1.81 & 0.00 & 28 & 100 \\
\hline & $>49$ & 90 & 0.8260 to 0.9553 & 64 & 0.5036 to 0.7664 & 2.53 & 0.15 & 36 & 97 \\
\hline & $>60.5$ & 40 & 0.3042 to 0.5105 & 93 & 0.8271 to 0.9802 & 5.66 & 0.64 & 55 & 88 \\
\hline & $>70.5$ & 12 & 0.05989 to 0.1997 & 98 & 0.9045 to 0.9995 & 6.54 & 0.90 & 59 & 84 \\
\hline \multirow[t]{6}{*}{ Cancer ratio } & $>5.61$ & 100 & 0.9615 to 1.000 & 27 & 0.1583 to 0.4030 & 1.37 & 0.00 & 23 & 100 \\
\hline & $>7.93$ & 90 & 0.8260 to 0.9553 & 54 & 0.3974 to 0.6701 & 1.95 & 0.18 & 30 & 96 \\
\hline & $>9.89$ & 82 & 0.7263 to 0.8910 & 75 & 0.6163 to 0.8561 & 3.28 & 0.24 & 42 & 95 \\
\hline & $>13.39$ & 70 & 0.5990 to 0.7921 & 93 & 0.8271 to 0.9802 & 9.83 & 0.32 & 68 & 93 \\
\hline & $>16.02$ & 61 & 0.5002 to 0.7056 & 96 & 0.8769 to 0.9956 & 16.99 & 0.41 & 79 & 92 \\
\hline & $>18.26$ & 47 & 0.3644 to 0.5739 & 100 & 0.9362 to 1.000 & & 0.53 & 100 & 90 \\
\hline
\end{tabular}

$L R+$ positive likelihood ratio, $L R-$ negative likelihood ratio, $P P V$ positive predictive value, $N P V$ negative predictive value, $C I$ confidence interval 
of pleural lymphocytosis especially when $80 \%$ of lymphocyte is chosen as the reference value [22]. The possible mechanism of TPE is the interaction between Mycobacterium tuberculosis and the immune system, causing hypersensitivity reaction to mycobacterial proteins in the pleura [14]. This forms the basis of the study due to the reciprocal change seen between pleural lymphocyte count and serum LDH and pleural ADA in MPE. The incidence of neutrophil predominance in MPE is low about 8\% [23].

Regarding the median value of the cancer ratio, it was higher in MPE (median/range; 18.03/10.87-29.73) than TPE (median/range; 6.1/4.46-9.17) and PPE (median/ range; $8.7 / 6.79-11.79)$ groups with statistically significant difference $(P<0.001)$, and this can be explained by the higher value of LDH in serum and lower value of ADA in pleural fluid (the components used to calculate the cancer ratio) in the MPE than TPE and PPE groups. Verma et. al. [7] reported that serum LDH and cancer ratio can be used for differentiation between malignant and non-malignant effusion and used a cutoff level of > 20 as highly predictive of MPE (whether lymphocytic or neutrophilic). Again, Verma et. al. [8] mentioned that MPE showed significantly higher cancer ratio in comparison with TPE. Combining the biomarkers to improve the accuracy of tests in diagnosing pleural effusion is not a novel idea. Diacon et al. [24] showed an increased ADA specificity to $100 \%$ when combined with pleural lymphocyte to neutrophil ratio (L:N ratio), in comparison with $95 \%$ when used alone for diagnosing TPE. Also, Burgess et al. [25] demonstrated improvement in specificity of ADA from 81 to $95 \%$ by combining it with L:N ratio for diagnosing TPE.

In the present study, the cancer ratio plus showed statistically significant difference between MPE (median/ range; 40.13/24.68-60.88) and TPE (median/range; 8.06/ 5.22-11.14) ( $\mathrm{P} 1<0.001)$, and this can be explained by the higher level of cancer ratio and the relative lower level of pleural lymphocyte count (the components used to calculate the cancer ratio plus) in MPE in comparison with TPE. The cancer ratio plus was also high in PPE (median/range; 47.5/32.15-68.78) with the difference between TPE and PPE in cancer ratio plus also statistically significant $(\mathrm{P} 3<0.001)$ due to the very low level of lymphocyte count in PPE in relation to TPE, while the difference between MPE and PPE was statistically insignificant $(\mathrm{P} 2=0.5)$. So cancer ratio is more appropriate in predicting MPE than cancer ratio plus. In contrast, Verma et. al. [8] reported that the ROC-derived cutoff level of "cancer ratio" allowed differentiating MPE from TPE with sensitivity and specificity of 0.95 and 0.85 and mentioned that the cutoff level of "cancer ratio plus" of $>30$ improved the sensitivity and specificity to 0.97 and 0.94 , respectively. The positive likelihood ratio (PLR) at this cutoff level was 41 , while the negative likelihood ratio (NLR) was found to be 0.06 . PLR value of 41 signifies that patients with cancer have about 41-fold higher chance of having "cancer ratio plus" of $>30$ compared with patients without cancer. This makes the effusion very likely to be malignant with a very high probability. Also, they mentioned that NLR at this cutoff was found to be 0.06 which suggests that if the "cancer ratio plus" is $<30$, the probability that this patient has cancer is $6 \%$, which is low enough to make the diagnosis of cancer highly unlikely.

Serum LDH and pleural fluid lymphocyte count ratio was statistically significant between our study groups $(P$ $<0.001$ ) with higher values in MPE (median/range; 700/ 506.8-950.4) and PPE (median/range; 1200/762.121754.54) than in TPE (median/range; 271.11/216.49332.5), and this can be explained by the high serum LDH level and lower pleural fluid lymphocyte count in MPE in relation to TPE. Also, PPE is mainly neutrophilic effusion. Multiple logistic regression analysis that was adjusted using stepwise elimination methods for all predictors of malignant effusion pointed on age, "cancer ratio," "serum LDH to pleural lymphocyte count" as significant positive predictors of MPE with $P$ values < $0.001,0.007$, and 0.003 respectively (Table 3 ). This again agrees with Verma et. al. [8] who mentioned that serum LDH to pleural fluid lymphocyte count ratio was significantly higher in MPE and can be used as a discriminating predictor between malignant and $\mathrm{TB}$ pleural effusion, but they reported that the obtained sensitivity and specificity from the ROC-derived cutoff level of > 800 were 0.63 and 0.85 , respectively. These were less than that of "cancer ratio" and "cancer ratio plus." Also, the AUC of 0.68 suggests that this ratio may not be useful in clinical practice. This goes hand in hand with our results that revealed that the AUC of cancer ratio and age suggests good accuracy ( $88 \%$ and $84 \%$ respectively) with $P<0.001$ for both, while AUC for "serum LDH to pleural fluid lymphocyte ratio" was fair to be accepted (65\%) and $P$ value was 0.001 (Fig. 1). Also, the comparison between the AUC of significant predictors showed that the difference of area between "cancer ratio" and "serum LDH to pleural fluid lymphocyte ratio" was significant, the same for the difference of area between age and serum LDH to pleural fluid lymphocyte count ratio $(P<0.0001$ and 0.0003 respectively) (Table 4$)$.

Cancer ratio showed a significantly higher value in MPE patients in particular at a cutoff point of $>16.02$ with sensitivity of $61 \%$, specificity of $96 \%$, PPV of $79 \%$, and NPV of $92 \%$. The positive likelihood ratio $(\mathrm{LR}+)$ value was 16.99 , while the negative likelihood ratio (LR-) at this cutoff was found to be 0.41 . Also, age is predictive of malignant pleural effusion at a cutoff point of $>60.5$ with sensitivity of $40 \%$, specificity of $93 \%$, PPV of $55 \%$, and NPV of $88 \%$ (Table 5). To determine the cutoff value, a compromise between 
sensitivity and specificity is required [26]. The high sensitivity makes the test good for screening, but a highly sensitive test has a greater tendency to give a high number of falsepositive results which may give false alarm for malignancy and mental agony. A highly specific test gives more definitive diagnosis, so we focused on high specificity with reasonable sensitivity. The cutoff $>16.02$ was considered as the best cutoff for cancer ratio as the LR- (negative likelihood ratio) was 0.41 at this cutoff and LR+ (positive likelihood ratio) value was 16.99 which suggests that malignant patients have about 16-fold higher chance of having cancer ratio (serum LDH to pleural fluid ADA ratio) of > 16.99 compared with patients without cancer. This high probability would be considered high enough to consider an effusion very likely to be malignant. On the other hand, NLR at this cutoff was found to be 0.41 which suggests that if the cancer ratio is $<16.99$, the probability that this patient had malignancy is low enough to make the diagnosis of MPE unlikely.

\section{Conclusion}

This study described the ability of simple biomarkers such as LDH in serum, ADA in pleural fluid, and lymphocyte count in diagnosis of malignant pleural effusion. These findings can help in rapid screening of patients with malignant pleural effusion in a simpler manner, with no added cost or test. Cancer ratio was found to be helpful in detecting MPE especially in patients with negative pleural fluid cytology. Cancer ratio can be used as a guide in selecting patients in whom to search for malignancy more actively. Also, it may help in the identification of patients who may need pleural biopsy to confirm the diagnosis of malignancy (cancer ratio $>16.02$ ). This study included prospective data which was consistent with previous reports from other studies. The limitations of this study include the following: (1) other causes of lymphocytic exudative effusions such as connective tissue diseases were not included in the study to validate these results in this group of patients, and (2) most patients with malignant pleural effusion had lung cancer with few numbers of lymphoma cases and extra thoracic malignancies. MPE associated with lymphoma can also have high levels of ADA and mimic TPE, so further studies including larger number of patients with MPE from lymphoma are required. But MPE associated with lymphoma is rare in comparison with TPE and other causes of MPE.

\footnotetext{
Abbreviations

MPE: Malignant pleural effusion; LDH: Lactate dehydrogenase; ADA: Adenosine deaminase; TPE: Tuberculous pleural effusion; PPE: Parapneumonic effusions; TB: Tuberculosis; CR: Cancer ratio; ATP: Adenosine triphosphate; PLR: Positive likelihood ratio; NLR: Negative likelihood ratio; LR+: Positive likelihood ratio; LR-: Negative likelihood ratio; PPV: Positive predictive value; NPV: Negative predictive value
}

\section{Acknowledgements}

The authors thank all patients for the use of their data in this research. The authors also thank their colleagues in the Chest Diseases Departments, Cairo and Menoufia Universities, for their assistance.

\begin{abstract}
Authors' contributions
All authors have contributed actively in the work, and the manuscript has been read and approved by all the authors for submission. SA Hussein: study conception and design, performing thoracentesis and pleural biopsy, data acquisition and analysis, manuscript drafting, revision, and approval of the final manuscript. MY Elhefnawy: study conception or design, data acquisition and interpretation, performing thoracentesis and pleural biopsy, manuscript drafting, revision, and final approval.
\end{abstract}

\section{Funding}

This research did not receive any specific grant from funding agencies in the public, commercial, or not-for-profit sectors.

\section{Availability of data and materials}

The data used and/or analyzed during the current study are available from the corresponding author on reasonable request.

\section{Ethics approval and consent to participate}

Institutional research ethics committee has approved this study, but the reference number is not applicable and not available. Written informed consent was obtained from all study participants before enrollment in the study.

\section{Consent for publication}

A written informed consent was obtained from the patients for the publication of their details.

\section{Competing interests}

The authors declare no financial or non-financial competing interest.

\section{Author details}

${ }^{1}$ Faculty of Medicine, Cairo University, Cairo, Egypt. ${ }^{2}$ Faculty of Medicine, Menoufia University, Menoufia, Egypt.

Received: 31 March 2020 Accepted: 8 April 2020

Published online: 24 June 2020

\section{References}

1. Korczyńs ki P, Mierzejewski M, Krenke1 R, Safianowska A, Light RW (2018) Cancer ratio and other new parameters for differentiation between malignant and nonmalignant pleural effusions. POLISH ARCHIVES OF INTERNAL MEDICINE. 128 (6).

2. Porcel JM, Light RW (2006) Diagnostic approach to pleural effusion in adults. Am Fam Physician. 73:1211-1220

3. Davies HE, Davies RJO, Davies CWH (2010) Management of pleural infection in adults: British Thoracic Society pleural disease guideline. Thorax. 65 (2): ii41-ii53.

4. Solooki M. Diagnostic yield of cytology in malignant pleural effusion: impact of volume and repeated thoracentesis. Eur Respir J. 2011; 38 (Suppl 55): p3550.

5. Korczynski P, Krenke R, Safianowska A et al (2009) Diagnostic utility of pleural fluid and serum markers in differentiation between malignant and non-malignant pleural effusions. Eur J Med Res. 14(Suppl 4):128-133

6. Safianowska A, Krenke R, Dmowska-Sobstyl B et al (2006) Adenosine deaminase activity in tuberculous and malignant pleural effusions. Pneumonol Alergol Pol. 74:5-9

7. Verma A, Abisheganaden J, Light RW (2016) Identifying malignant pleural effusion by a cancer ratio (serum LDH: pleural fluid ADA ratio). Lung. 194: 147-153

8. Verma A, Dagaonkar RS, Marshall D, Abisheganaden J, Light RW (2016) Differentiating malignant from tubercular pleural effusion by cancer ratio plus (cancer ratio: pleural lymphocyte count). Can Respir J. 2:1-6

9. Light RW, Macgregor Ml, Luchsinger PC, Ball WC (1972) Pleural effusions: the diagnostic separation of transudates and exudates. Ann Intern Med. 77:507513 
10. Terpos E, Katodritou E, Roussou M, Pouli A, Michalis E, Delimpasi S, Parcharidou A, Kartasis Z, Zomas A, Symeonidis A, Viniou NA Anagnostopoulos N, Economopoulos T, Zervas K (2010) Dimopoulos MA; Greek Myeloma Study Group. Greece. Eur J Haematol. 85(2):114-119

11. Pfeiffer T, Schuster S, Bonhoeffer S (2001) Cooperation and competition in the evolution of ATP-producing pathways. Science. 292:504-507

12. Goldman Robert D, Kaplan Nathan O, Hall TC (1964) Lactic dehydrogenase in human neoplastic tissues. Cancer Res. 24:389-399

13. Porcel JM, Esquerda A, Bielsa S (2010) Diagnostic performance of adenosine deaminase activity in pleural fluid: a single-center experience with over 2100 consecutive patients. Eur J Intern Med. 21:419-423

14. Vorster MJ, Allwood BW, Diacon AH, Koegelenberg CFN (2015) Tuberculous pleural effusions: advances and controversies. Journal of Thoracic Disease. 7(6):981-991

15. Seibert AF, Haynes J, Middleton R, Bass JB (1991) Tuberculous pleural effusion: twenty-year experience. Chest. 99(4):883-886

16. Epstein DM, Kline LR, Albelda SM, Miller WT (1987) Tuberculous pleural effusions. Chest. 91(1):106-109

17. Berger HW, Mejia E (1973) Tuberculous pleurisy. Chest. 63(1):88-92

18. Vald'es L, Alvarez D, San Jos'e E, Penela P, Valle J, García-Pazos J, Suárez J, Pose A (1998) Tuberculous pleurisy. A study of 254 patients," Archives of Internal Medicine. 158(18): 2017-2021.

19. Ruan SY, Chuang YC, Wang JY, Lin JW, Chien JY, Huang CT, Kuo YW, Lee LN, Yu CJ (2012) Revisiting tuberculous pleurisy: pleural fluid characteristics and diagnostic yield of mycobacterial culture in an endemic area. Thorax. 67(9): 822-827

20. Light RW. "Tuberculous pleural effusions," in Pleural Diseases, R.W. Light, Ed., pp. 154-166, Williams \&Wilkinson, Baltimore, Md, USA, 3rd edition, 1995.

21. Sahn SA (1995) The diagnostic value of pleural fluid analysis. Seminars in Respiratory and Critical Care Medicine. 16(4):269-278

22. Frank W (2013) "Tuberculous pleural effusion" in Tuberculosis - Current Issues in Diagnosis and Management. 267-292.

23. Kushwaha R, Shashikala P, Hiremath S, Basavaraj HG (2008) Cells in pleural fluid and their value in differential diagnosis. Journal of Cytology. 25(4):138-143

24. Diacon AH, Van de Wal BW, Wyser C, Smedema JP, Bezuidenhout J, Bolliger CT, Walzl G (2003) Diagnostic tools in tuberculous pleurisy: a direct comparative study. European Respiratory Journal. 22(4):589-591

25. Burgess L, Maritz FJ, Le Roux I, Taljaard JJF (1996) Combined use of pleural adenosine deaminase with lymphocyte/neutrophil ratio: increased specificity for the diagnosis of tuberculous pleuritis. Chest. 109(2):414-419

26. Florkowski CM (2008) Sensitivity, specificity, receiver-operating characteristic (ROC) curves and likelihood ratios: communicating the performance of diagnostic tests. Clin Biochem Rev. 29(Suppl I): S83.

\section{Publisher's Note}

Springer Nature remains neutral with regard to jurisdictional claims in published maps and institutional affiliations.

\section{Submit your manuscript to a SpringerOpen ${ }^{\circ}$ journal and benefit from:}

- Convenient online submission

- Rigorous peer review

- Open access: articles freely available online

- High visibility within the field

- Retaining the copyright to your article

Submit your next manuscript at $\boldsymbol{\nabla}$ springeropen.com 